\title{
Lethal Encephalopathy in an Infant with Hypophosphatasia despite Enzyme Replacement Therapy
}

\author{
Adalbert Raimann ${ }^{\mathrm{a}, \mathrm{b}} \quad$ Christine Haberler ${ }^{\mathrm{c}}$ Janina Patsch ${ }^{\mathrm{b}, \mathrm{d}}$ \\ Diana-Alexandra Ertl ${ }^{a, b} \quad K^{2}$ ambis Sadeghi ${ }^{a} \quad$ Michael Freilinger $^{a} \quad$ Susanna Lang ${ }^{e}$ \\ Maria Schmook ${ }^{d}$ Barbara Plecko $^{f}$ Gabriele Haeusler ${ }^{a}$ b

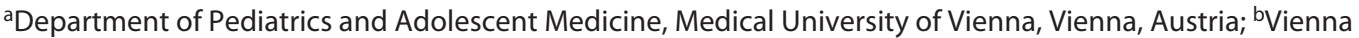 \\ Bone and Growth Center, Vienna, Austria; 'Institute of Neurology, Medical University of Vienna, Vienna, Austria; \\ 'Department of Biomedical Imaging and Image-Guided Therapy, Medical University of Vienna, Vienna, Austria; \\ eDepartment of Pathology, Medical University of Vienna, Vienna, Austria; $f^{f}$ Department of Pediatrics and Adolescent \\ Medicine, Medical University of Graz, Graz, Austria
}

\section{Established Facts}

- Morbidity and mortality in hypophosphatasia (HPP) are associated with residual alkaline phosphatase activity and skeletal manifestations.

- Pyridoxine-responsive seizures due to impaired cerebral passage are commonly discussed as most severe CNS manifestations in HPP.

- Effects of enzyme replacement therapy on neurologic symptoms are uncertain.

\section{Novel Insights}

- Progression of hypophosphatasia (HPP)-associated encephalopathy can occur even with early onset enzyme replacement therapy.

- HPP-associated encephalopathy due to neuronal ALP deficiency may occur despite pyridoxine responsiveness of neonatal seizures.

- Monitoring of neurological pathologies including encephalopathy has to be implemented for patients with HPP (at risk).

\section{Keywords}

Alkaline phosphatase · Asfotase alfa · Bone ·

Encephalopathy · Enzyme replacement therapy ·

Hypophosphatasia · Pyridoxine - Rare diseases · Vitamin B6

\begin{abstract}
Hypophosphatasia (HPP) is an inborn error of metabolism caused by loss-of-function mutations in the biomineralization-associated alkaline phosphatase gene, encoding tissuenonspecific alkaline phosphatase (TNSALP). Symptoms include skeletal hypomineralization and extra-skeletal mani-
\end{abstract}

(C) 2021 The Author(s)

Published by S. Karger AG, Basel

This article is licensed under the Creative Commons Attribution 4.0 International License (CC BY) (http://www.karger.com/Services/ OpenAccessLicense). Usage, derivative works and distribution are permitted provided that proper credit is given to the author and the original publisher.
Correspondence to:

Gabriele Haeusler, gabriele.haeusler@meduniwien.ac.at 
festations such as pyridoxine (B6)-responsive seizures due to impaired cerebral B6 passage. Since the introduction of enzyme replacement therapy (ERT), skeletal manifestations and B6-responsive seizures were reported to improve significantly. Nevertheless, there is an increasing evidence of B6-independent neurological manifestation of HPP including HPP-associated encephalopathy. Here, we present for the first time the brain alterations of an infant with neonatal HPP who died of neurological complications at the age of 5 months despite early initiation of ERT. CSF analysis showed normal concentrations of biogenic amines reflecting sufficient intracellular B6 availability. Postmortem histopathology revealed severe, localized affection of the cerebral cortex including cortical lesions in layers 2 and 3 in direct proximity to TNSALP-expressing neurons and hippocampal sclerosis. Our findings confirm that TNSALP deficiency may lead to a severe encephalopathy. We hypothesize that HPP-associated encephalopathy resistant to currently available ERT may develop in addition and probably independently of typical B6-responsive seizures in some patients. Prospective, controlled studies with close neurological follow-up including brain imaging are needed to identify patients at risk for severe neurological symptoms despite ERT.

(C) 2021 The Author(s)

Published by S. Karger AG, Basel

\section{Introduction}

Hypophosphatasia (HPP) is a rare metabolic disorder caused by inactivating mutations in the biomineralization-associated alkaline phosphatase (ALPL), a gene encoding tissue-nonspecific alkaline phosphatase (TNSALP) [1]. The survival and prognosis for patients with life-threatening neonatal HPP have significantly improved since the introduction of enzyme replacement therapy (ERT) with asfotase alpha [2]. Moreover, struc-

Fig. 1. Comparison of MR images at the age of 4 days (left) and 2.5 weeks (right) before death. Coronal T2-TSE images show a significant brain atrophy with enlarged ventricles and widening of the subarachnoid space at the age of 4.5 months, 2.5 weeks before death, (b) compared with the postnatal image (a). Axial diffusionweighted images reveal bilateral diffusion restriction at the subependymal border of the head of the caudate nucleus (d, arrows) (with correspondingly decreased ADC values [not shown]), adjacent to the third ventricle (f, arrows) and within the hippocampi (f, h, arrows) which were not present at birth (c, e, g). Axial T1weighted gradient-echo images show regularly progressing myelination of the internal capsule and the splenium of the corpus callosum corresponding to the age of the patient $(\mathbf{j})$ compared with the postnatal MRI (i).

Hypophosphatasia-Associated Encephalopathy
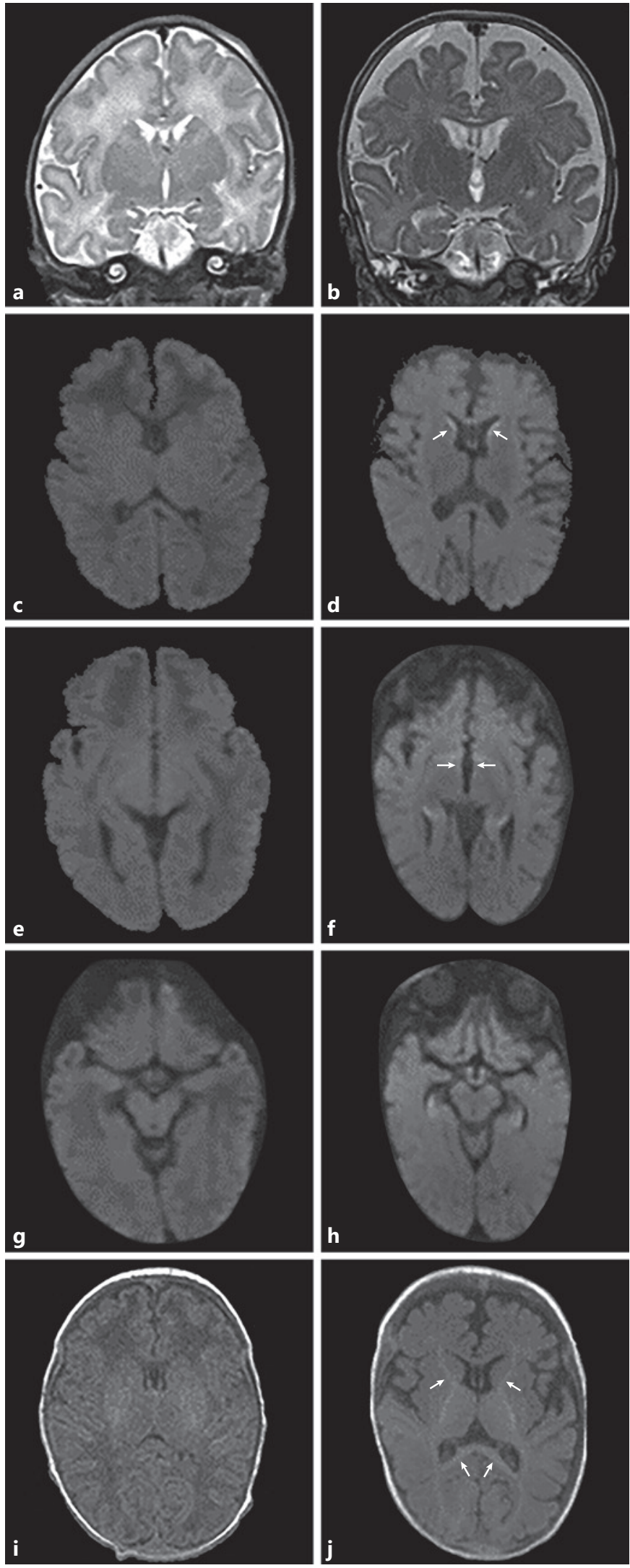

Horm Res Paediatr 2021;94:390-398 DOI: $10.1159 / 000520341$ 

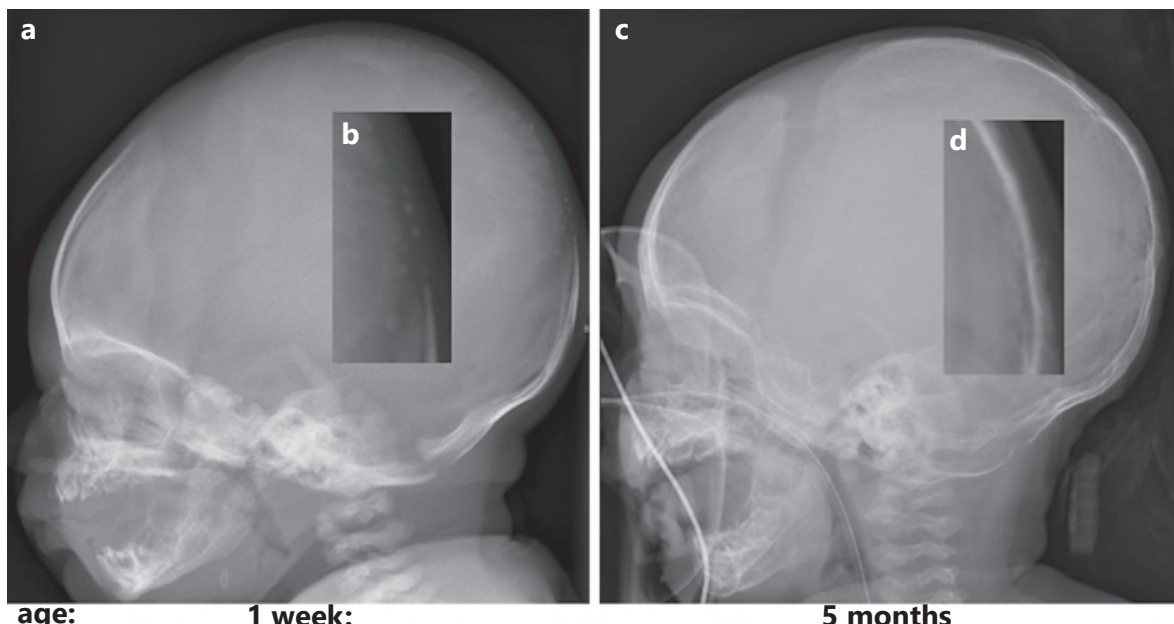

Fig. 2. Radiographic assessment. At presentation $(\mathbf{a}-\mathbf{h})$, only a mild skeletal phenotype was observed with delayed ossification of the skull $(\mathbf{a}, \mathbf{b})$ and impression of mild tongue-like lucencies at the lower metaphyses of femur and upper metaphysis of tibia but otherwise absent signs of demineralization (e), rickets or fractures $(\mathbf{f}-\mathbf{h})$. Follow-up imaging at 5 months of age revealed improved mineralization/ossification of the skull; $(\mathbf{c}, \mathbf{d})$ but otherwise severe global bone loss (i-I) and multiple spine fractures $(\mathbf{k})$.
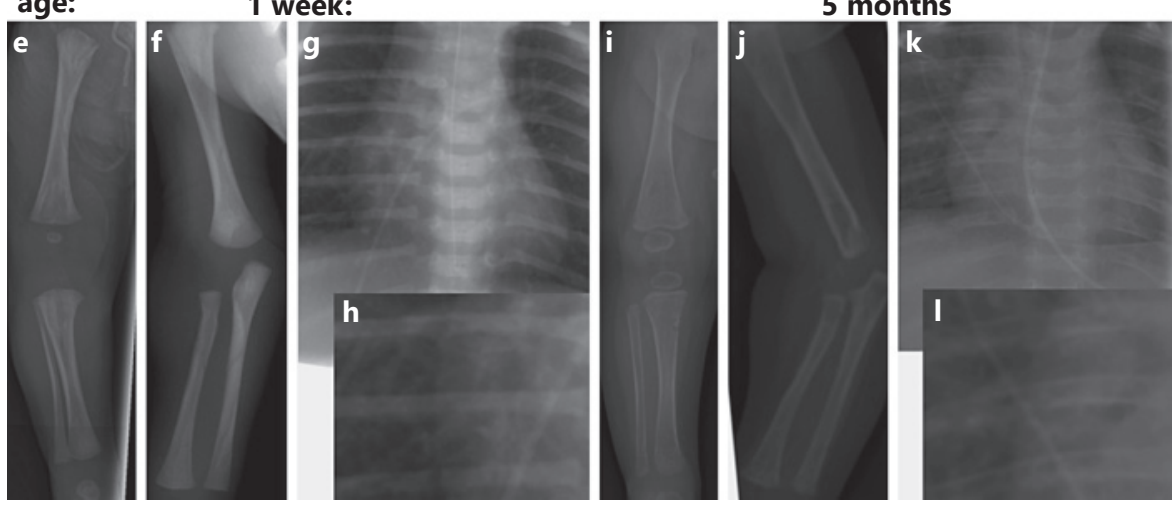

tural brain anomalies and a poorly understood form of encephalopathy have been reported in single patients with neonatal HPP as well as in animal models [3-5].

Here, we present the case of a child with neonatal pyridoxine (PN)-responsive seizures, compound heterozygous genotype, and lethal outcome at the age of 5 months despite start of therapy with recombinant, bone-targeted ERT during the first week of life. We report for the first time histopathological changes of the brain in human HPP in context with a detailed analysis of the clinical course during neurological deterioration. Our findings are in line with a severe encephalopathy, presumably on the basis of cerebral TNSALP deficiency despite early ERT.

\section{Case Report/Case Presentation}

\section{Clinical Course}

The male index patient was delivered at term after an uneventful pregnancy and was the first child of nonconsanguineous, Caucasian parents. During the first $48 \mathrm{~h}$, the neonate showed hyperexcitability and had 2 tonic-clonic seizures, which ceased after a single intramuscular dose of $50 \mathrm{mg}$ PN hydrochloride (PN-HCl). EEG and cra- nial MRI on the second day of life showed no pathologies (Fig. 1). Laboratory investigation revealed undetectable levels of serum alkaline phosphatase (ALP). Genetic analysis confirmed the diagnosis of HPP by detection of compound heterozygosity for ALPL (c.667C $>\mathrm{T}$, c.984_986delCTT). Both mutations have been found as homozygous alleles in neonatal HPP [6, 7]. ERT (asfotase alpha, Strensiq@), $1 \mathrm{mg} / \mathrm{kg}$ subcutaneously 6 days/week) was initiated on day 6 . Mild skeletal hypomineralization of the skull (Fig. 2) was observed on plain radiographs, but the axial and appendicular skeleton appeared normal. The child was discharged after 3 weeks with mild muscular hypotonia for which he received physiotherapy and was seen for regular neurological follow-up. Adhesion to treatment was excellent documented by a rise of serum ALP to supraphysiologic levels.

At 12 weeks of age, the patient developed dyskinesia of the face and upper extremities, which ceased after intramuscular application of $12.5 \mathrm{mg} \mathrm{PN}-\mathrm{HCl}$ followed by low-dose oral PN-HCl supplementation ( $5 \mathrm{mg}$ /day). He showed reduced alertness and muscular hypotonia which resolved within a few days. Repeatedly, EEGs were normal.

At the age of 4.5 months, while still on PN-HCl, $5 \mathrm{mg} / \mathrm{day}$ orally, the boy was readmitted with colic-like pain episodes and intermittent strabismus. Arterial hypertension was noted, and apnea necessitated a transfer to the pediatric intensive care unit and mechanical ventilation. cMRI showed marked brain atrophy and impaired periventricular diffusion (Fig. 1b). EEG for the first time showed a spike and wave pattern over the left frontal region. The ERT dose was doubled $(2 \mathrm{mg} / \mathrm{kg})$ and switched to a daily regime. 
Table 1. Analysis of neurotransmitters and PN derivates in CSF/ peripheral blood at day 144

\begin{tabular}{|c|c|c|c|}
\hline \multicolumn{4}{|c|}{ Age at sample acquisition: $149 \mathrm{~d}$} \\
\hline \multirow{2}{*}{$\begin{array}{l}\text { Specific therapy, } \\
\mathrm{mg} / \mathrm{kgBW} / \text { day }\end{array}$} & & asfotase alfa & $2 \mathrm{mg} / \mathrm{kg}$ \\
\hline & & $\mathrm{PN}-\mathrm{HCl}$ & $30 \mathrm{mg} / \mathrm{kg}$ \\
\hline \multirow{2}{*}{\multicolumn{4}{|c|}{$\begin{array}{c}\text { B6 derivates } \\
\text { CSF }\end{array}$}} \\
\hline & & & \\
\hline PLP & 1,540 & $\mathrm{nmol} / \mathrm{L}$ & $<60$ \\
\hline $\mathrm{PL}$ & 2,310 & $\mathrm{nmol} / \mathrm{L}$ & $70-83$ \\
\hline PM & 24 & $\mathrm{nmol} / \mathrm{L}$ & $<4$ \\
\hline PN & 1,430 & $\mathrm{nmol} / \mathrm{L}$ & $<1$ \\
\hline PA & 172 & $\mathrm{nmol} / \mathrm{L}$ & $<2$ \\
\hline \multicolumn{4}{|l|}{ Serum } \\
\hline MMA & 759 & $\mathrm{nmol} / \mathrm{L}$ & $73-271$ \\
\hline PLP & 5,010 & $\mathrm{nmol} / \mathrm{L}$ & $10-289$ \\
\hline$P L$ & 5,830 & $\mathrm{nmol} / \mathrm{L}$ & $0.4-85$ \\
\hline PM & 28 & $\mathrm{nmol} / \mathrm{L}$ & $<1$ \\
\hline PN & 19,600 & $\mathrm{nmol} / \mathrm{L}$ & $<1$ \\
\hline PA & 5,900 & $\mathrm{nmol} / \mathrm{L}$ & $4-584$ \\
\hline \multicolumn{4}{|l|}{ Biogenic amines } \\
\hline \multicolumn{4}{|l|}{ CSF } \\
\hline 5-HIAA & 261 & $\mathrm{nmol} / \mathrm{L}$ & $150-800$ \\
\hline HVA & 671 & $\mathrm{nmol} / \mathrm{L}$ & $310-1,100$ \\
\hline $\mathrm{HVA} / 5-\mathrm{HIAA}$ & 2.6 & & - \\
\hline DOPAC & 31 & $\mathrm{nmol} / \mathrm{L}$ & - \\
\hline MHPG & 77 & $\mathrm{nmol} / \mathrm{L}$ & $98-188$ \\
\hline L-DOPA & 8.2 & $\mathrm{nmol} / \mathrm{L}$ & $<25$ \\
\hline 3-OMD & 25.3 & $\mathrm{nmol} / \mathrm{L}$ & $<300$ \\
\hline $5 \mathrm{OH}$ Trp & 11.5 & $\mathrm{nmol} / \mathrm{L}$ & $<10$ \\
\hline 5MTHF & 260 & $\mathrm{nmol} / \mathrm{L}$ & $64-182$ \\
\hline Neopterin & 24 & $\mathrm{nmol} / \mathrm{L}$ & $15-35$ \\
\hline Biopterin & 18.2 & $\mathrm{nmol} / \mathrm{L}$ & $20-70$ \\
\hline
\end{tabular}

Vitamin B6 vitamers were elevated in both plasma and CSF. Analysis of biogenic amines was without pathologic findings. $\mathrm{PL}$, pyridoxal; $\mathrm{PM}$, pyridoxamine; $\mathrm{PN}$, pyridoxine; $\mathrm{PA}$, pyridoxic acid; PLP, pyridoxal 5'-phosphate; MMA, methylmalonic acid; 5-HIAA, 5-hydroxyindoleacetic acid; HVA, homovanillic acid; DOPAC, 3,4-dihydroxyphenylacetic acid; MHPG, 3-methoxy-4-hydroxyphenylglycol; L-DOPA, L-dihydroxyphenylalanine; 3-OMD, 3-O-methyldopa; 5 OH Trp, L-5-hydroxytryptophan; 5MTHF, L-5-methyltetrahydrofolate; $\mathrm{PN}-\mathrm{HCL}, \mathrm{PN}$ hydrochloride.

$\mathrm{PN}-\mathrm{HCl}$ was increased to $40 \mathrm{mg} / \mathrm{kg} /$ day intravenously and due to lack of clinical improvement switched to oral pyridoxal 5 '-phosphate (PLP) $40 \mathrm{mg} / \mathrm{kg} /$ day after 1 week. In addition, magnesium gluconicum (700-2,100 mg/day) and calcium folinate therapy (3 $\mathrm{mg} / \mathrm{kg} /$ day) were started to optimize residual ALP activity and intracellular metabolism.

Cerebrospinal fluid CSF and plasma samples were taken at age 5 months, while the patient was on PN-HCl $30 \mathrm{mg} / \mathrm{kg} /$ day. Vitamin B6 vitamers were markedly elevated in plasma and CSF. Analysis of biogenic amines gave normal results (Table 1).

High-dose caffeine citrate and doxapram allowed extubation after 16 days, yet the patient required noninvasive ventilatory as-

Hypophosphatasia-Associated

Encephalopathy sistance. Following consensus between parents and medical staff, the patient was moved to palliative care and died at the age of 5.5 months 9 days after extubation due to respiratory failure.

\section{Postmortem Analysis of the Brain}

The brain was edematous and had a fresh weight of $666 \mathrm{~g}$ on macroscopic examination. The cerebral white matter appeared shrunken and grayish. The cerebral cortex, cerebellum, and brainstem showed no macroscopic abnormalities.

A mild to moderate spongiosis was seen on histopathological examination in the superficial cortical layers II and III but was less pronounced in layer III (Fig. 3a). Shrunken red neurons and occasional apoptotic features were present in layer II, and anti-HLADR staining revealed an increase in microglial cells (Fig. 3b). These changes were most pronounced in the frontal lobe, including the cingulate gyrus, and the temporal and parietal cortex. The mammillary bodies displayed a bilateral severe spongiosis and shrunken neurons accompanied by microglia activation (Fig. 3c, d) without bleeding or endothelial proliferations. Similar lesions were observed in a patchy pattern along the walls of the third and the lateral ventricles, especially close to the head of the caudate nucleus (Fig. 3e, f). The hippocampus showed a severe loss of neurons in the pyramidal layer within the sectors CA1 and CA4, and the subiculum, accompanied by a severe increase in microglial cells (Fig. 3i-k).

A prominent reactive astrogliosis could be observed in the cerebral white matter (Fig. 3g), whereas the myelination was appropriate for the patient's age (Fig. 3h). No lesions were detectable in the basal ganglia, thalamus, cerebellum, or brain stem.

\section{Postmortem Analysis of Bone}

Specimens from rib, femur, spine, and calvaria were taken and analyzed. Characteristic changes of HPP were found in the zone of enchondral ossification: the layer of columnar cartilage was lengthened with few blood vessels and ballooning of chondrocytes. A broad layer of disorganized and uncalcified osteoid with islands of growth cartilage was present at the border to the growth plate. Osteoblasts were rarely present.

\section{Discussion}

TNSALP is a membrane-bound ectophosphatase encoded by ALPL which is found in a vast range of skeletal and extra-skeletal tissues including the central nervous system (CNS) [8-10]. The diversity of tissue-specific substrates, such as pyrophosphates in mineralized tissues and pyridoxal (PL) in the CNS, explains the broad spectrum of symptoms in patients with TNSALP deficiency.

Vitamin B6-responsive seizures as observed in our patient have been described as a main neurological manifestation of neonatal HPP with a detrimental outcome and are probably caused by reduced PL passage over the blood-brain barrier as well as impaired cellular uptake into neurons $[1,11]$. In a subset of patients with severe HPP, neurologic symptoms may represent the main 

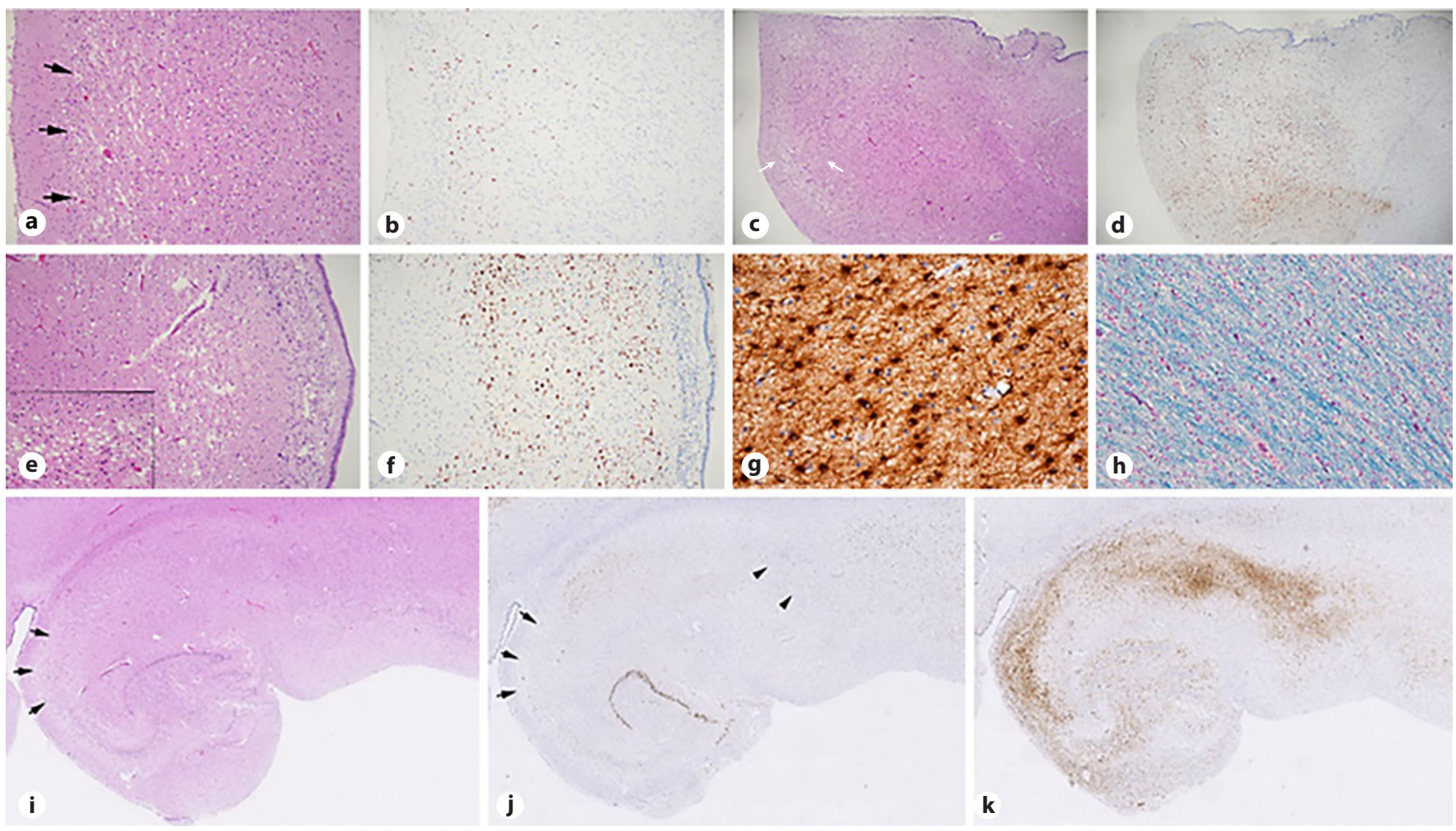

Fig. 3. Postmortem histopathological examination of the brain. Histopathological examination of the brain reveals spongiosis (a) and microglial activation in layer 2 (b), less pronounced in layer 3 , of the cerebral cortex. The mammillary bodies show severe spongiosis and shrunken neurons (c) and prominent microglial activation (d). A lesion subventricular close to the head of the caudate nucleus reveals spongiosis (e), shrunken neurons and apoptosis (e inset) as well as microglial activation (f). The cerebral white matter

shows a prominent gliosis $(\mathbf{g})$, whereas no defects are found in the myelination (h). The hippocampus is severely damaged with spongiosis and nerve cell loss in sectors CA1 and CA4 of the pyramidal layer (arrows $\mathbf{i}, \mathbf{j}$ ) and the subiculum (arrow heads $\mathbf{i}, \mathbf{j}$ ), which is confirmed by a concomitant microglial activation $(\mathbf{k})$. (a, c, e, i hematoxylin-eosin stain, $\mathbf{b}, \mathbf{d}, \mathbf{f}, \mathbf{k}$ HLADR immunohistochemistry, g, GFAP immunohistochemistry, Kluver-Barrera myelin stain; original magnification $\mathbf{a}-\mathbf{f}, \mathbf{h}: \times 4, \mathbf{g}: \times 40, \mathbf{h}: \times 10, \mathbf{i}-\mathbf{j}: \times 4)$. manifestation of disease (Table 2) and evidence of a severe HPP-associated, vitamin B6-independent encephalopathy has emerged [12-14].

In ALPL knockout mice (Akp2-/-), the effects on neural tube development were described as exceeding B6associated symptoms and accounting for compromised autonomic nervous system functions [3]. This accounts for the synaptic dysfunction and myelin abnormalities found in both spinal cord and cerebral cortex of Akp-/mice [15]. Recently, specific metabolic changes involving mainly inhibitory neurotransmitters (GABA, adenosine) and myelin synthesis (NAA, NAG) could be proven in Akp2-/- mice by MRI SPECT [16]. In vitro, TNSALP was found to play an essential role in principal functions such as cell proliferation and differentiation as well as in axonal development in hippocampal neurons in murinecultured multipotent neural stem cells $[17,18]$. Based on histopathological findings in Akp2-/- mice, a primary defect in neural tube development during embryonic life was speculated as the underlying mechanism [3]. This points to a neurologic phenotype beyond B6-dependent seizures with a possible onset during fetal development and a complex pathophysiology, including impaired inhibitory pathways as well as involvement of the limbic system.

Human HPP-associated encephalopathy has so far only been documented by MRI: a severe fatal multicystic encephalomalacia with band-like cortical necrosis has been reported in a child refractory to PN treatment [4]. A patient was found to have MRI alterations in the dorsolateral medulla oblongata at the age of 39 days and in the further course of disease a volume loss in the white matter and basal ganglia and a cystic degeneration of the white matter [19]. In contrast to these severe changes, reversible 


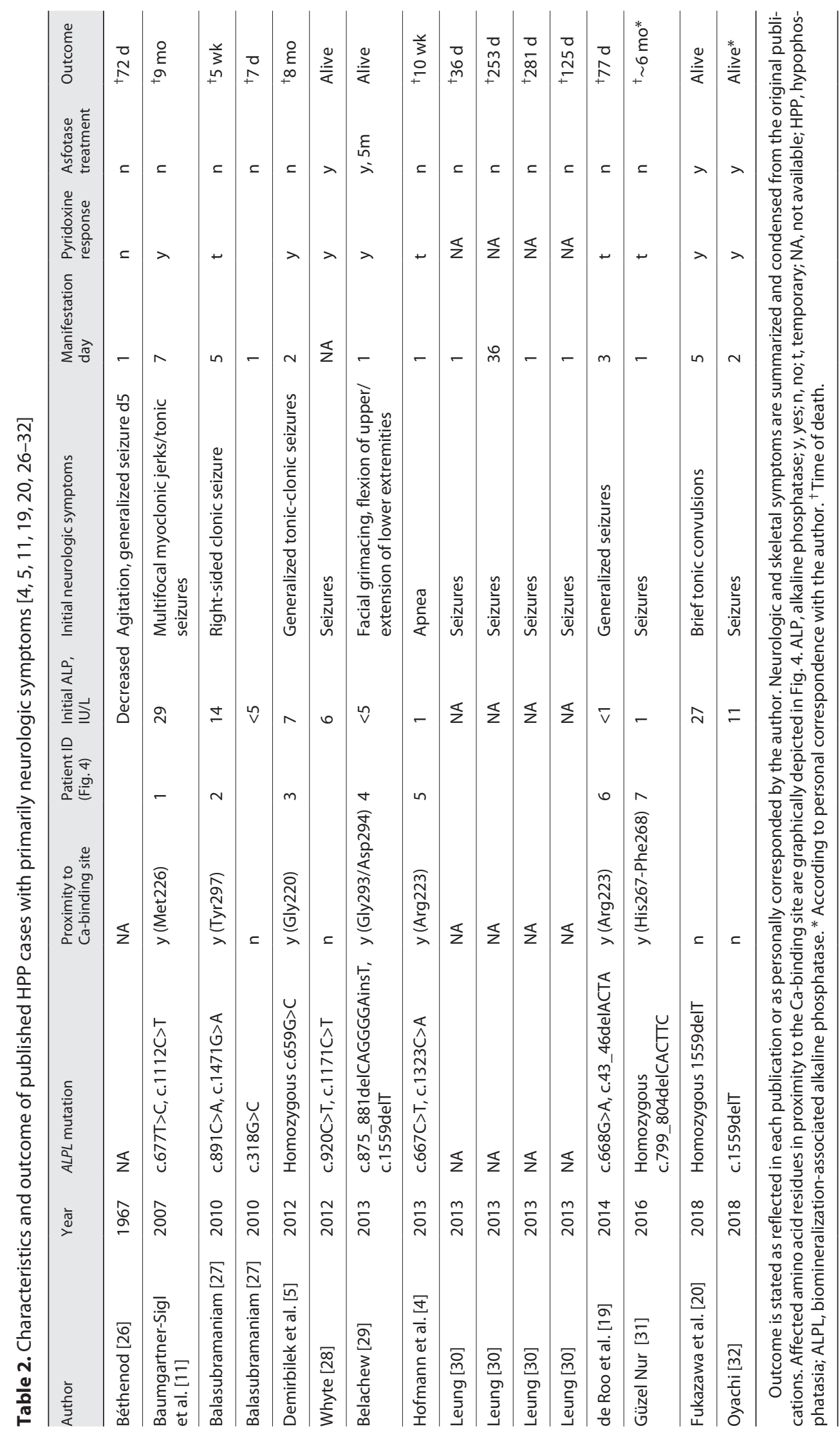


bilateral lesions in the dorsal brain stem including the medulla oblongata have been described [20]. MRI in our patient was unremarkable at day 4 , but showed a severe atrophy and signal hyperintensities periventricular and in the hippocampi at age 4.5 months. These periventricular changes could be confirmed upon postmortem histological analysis and showed similarities to Wernicke encephalopathy (thiamine deficiency). In our patient, white matter was gliotic without signs of myelin defects. Interestingly, we observed cortical lesions in layers 2 and 3 in direct proximity to TNSALP-expressing neurons in layer 4 as reported in human neocortex [21]. The changes in our patient were reminiscent of mitochondrial DNA depletion syndrome 4A (Alpers type). Hippocampal lesions are described in many inborn errors of metabolism closely related to the manifestation of epilepsy. A similar pattern of cortical lesions may be observed after hypoxicischemic brain damage, yet we can exclude hypoxemic episodes in our patient until his neurological deterioration at age 4.5 months, where MRI already demonstrated severe brain atrophy. Asfotase alfa was applied from first week of life at the maximum recommended dosage $(6 \mathrm{mg} /$ $\mathrm{kgBW}$ ) and further increased above recommendations $(12 \mathrm{mg} / \mathrm{kgBW})$ at first signs of EEG alterations.

In our patient, administration of high-dose $\mathrm{PN}-\mathrm{HCl}$ or PLP as the active form of vitamin B6 was clinically ineffective to arrest the progression of severe encephalopathy. Normal concentrations of biogenic amines in CSF reflect sufficient intracellular PLP availability as PLP is an important cofactor in the synthesis of these neurotransmitters. Incomplete rescue of the neurologic phenotype by vitamin $\mathrm{B} 6$ administration has also been observed in 2 Akp2-/- mouse strains, although to different extents: while $2 / 3$ of animals responded to treatment in the first mouse model, only temporary amelioration was observed in the second $[3,22]$.

So far, HPP-associated encephalopathy has only been described in severe neonatal forms of the disease with residual ALP enzyme activity below 10\% (Table 2). With such low residual activity, formation of PL is severely impaired, as illustrated by increased PLP concentrations in the plasma and CSF of patients with HPP before supplementation of B6. It has therefore been discussed if additional vitamin B6 supplementation may have a deleterious effect by increasing extracellular PLP concentrations to a toxic level [30] [23]. HPP-associated encephalopathy has been observed irrespective of vitamin B6 supplementation $[4,19]$ and at least 1 patient with higher residual ALP activity responded to increased doses of vitamin B6 treatment [20]. Still, excessive concentrations of PLP in the ex-

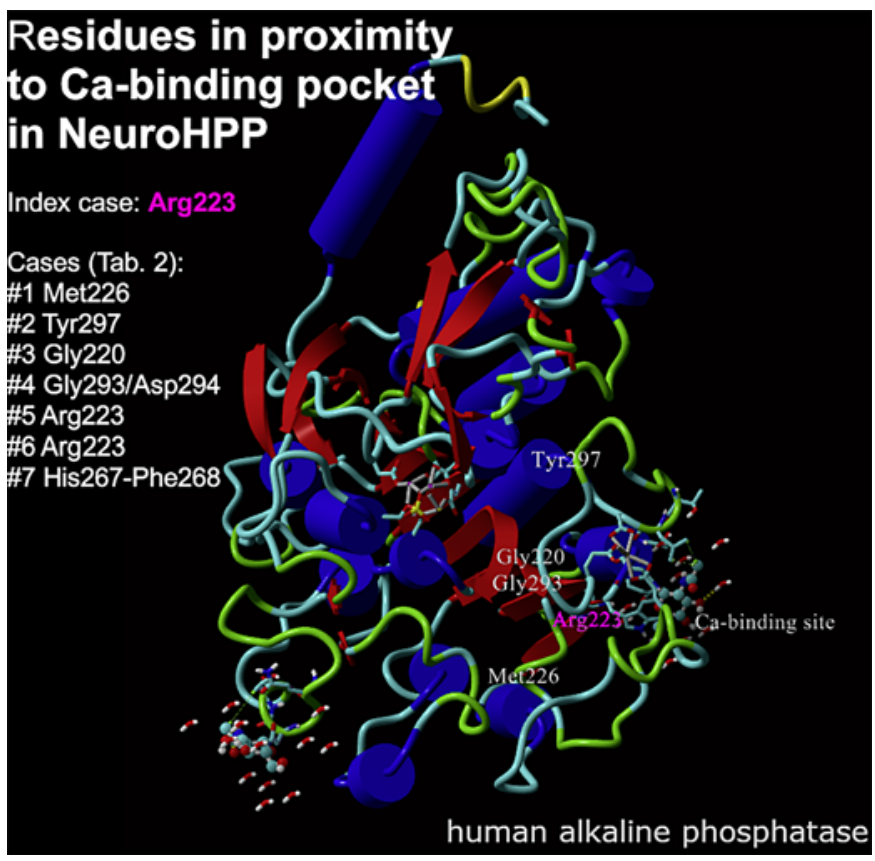

Fig. 4. $A L P L$ mutations of patients with primarily neurologic manifestation of HPP in proximity to the Ca-binding site. The majority of published cases with primarily neurologic symptoms of HPP feature inactivating mutations with low to undetectable residual ALP activity (Table 2). In 7/10 of cases with genetics available, mutations of ALPL in direct proximity to the Ca-binding pocket could be identified as depicted (ALPL monomer, Uniprot: 2GLQ). The affected residue of the reported patient Arg223 is localized in central proximity and labeled pink. Additionally, residue numbers of patients with mainly neuralgic HPP with mutations in proximity of this structural site is marked as listed in Table 2, column "Proximity to Ca-binding site": patient 1 Met226 (c.677T>C), patient 2 Tyr297 (c.891C>A), patient 3: Gly220 (c.659G>C), patient 4 Gly293/Asp294 (c.875_881delCAGGGGAinsT), patient 6 Arg223. HPP, hypophosphatasia; ALP, alkaline phosphatase; ALPL, biomineralization-associated alkaline phosphatase.

tracellular space cannot be excluded as a contributing factor in HPP-associated encephalopathy. Interestingly, our patient exhibited only mild features of mineralization deficits at birth despite severe ALP deficiency. While demineralization signs in radiographs occurred in the clinical course, the discrepancy between moderate skeletal affection and severe neurologic manifestation suggests specific pathomechanisms in these 2 organ systems with a certain degree of independent affection. Thus, a mild skeletal phenotype at birth does not rule out lethal HPP. In our case, the mild initial phenotype with continuous deterioration and missing data on neurologic complications under treatment posed/contributed to/an extremely difficult decision basis for caretakers and parents. 
Kinetic characterization of ALPL mutations revealed that some mutations were efficient in hydrolyzing inorganic pyrophosphate while inefficient in hydrolyzing PLP, which could explain why some patients with neonatal HPP manifest neurologic disease and others do not[24]. Interestingly, most patients with primarily neurologic manifestation carry mutations in proximity to the Ca-binding site of TNSALP, distant from the active site pocket (Fig. 4). An eventual genotype-phenotype association caused by substrate or tissue specificity remains to be determined.

Outcome data from patients with perinatal HPP and ERT are sparse. Our patient received ERT from a very early stage of disease. Like in other metabolic disorders, ERT in HPP is not expected to pass the blood-brain barrier and rescue a CNS disease. Nonbone targeted or intrathecal ERT may be needed to treat a CNS disease associated with neonatal HPP. While the postnatal impact of cerebral TNSALP deficiency might be positively addressed/influenced by such an option, developmental deficiencies, as observed in animal studies, might be irreversible. At the present state of therapeutic options, the aspects of clinical neurological development including HPP-associated encephalopathy have to be implemented in information provided to parents as well as current monitoring guidelines for children with severe HPP [25].

\section{Acknowledgments}

We gratefully acknowledge the parents of the patient for giving their consent for postmortem investigations. We also thank Deborah Mathis, University Children's Hospital Zurich, for investigating vitamin B6 vitamers; Benjamin Bausenhardt, University Hospital Tulln, for the perinatal management of the patient; and Elise Langdon-Neuner for proofreading the manuscript.

\section{Statement of Ethics}

This study protocol was reviewed, and the need for approval was waived by the Ethical Committee of the Medical University of Vienna, as published research complies with the guidelines for human studies in accordance with the World Medical Association Declaration of Helsinki. Written informed consent to perform autopsy and for publication of their clinical details and/or clinical images was obtained from the parents of the patient.

\section{Conflict of Interest Statement}

The authors have no conflicts of interest to declare.

\section{Funding Sources}

There is no external funding relevant to the study, preparation of data, or the manuscript.

\section{Author Contributions}

Conception and design of the work were conceived by A.R., D.A.E., C.H., M.S., B.P., and G.H. Acquisition of data was performed by A.R., J.P., D.A.E., K.S., M.F., S.L., M.S., and G.H. Data were analyzed by A.R., C.H., J.P., D.A.E., M.F., S.L., M.S., and G.H. Interpretation of data for the work was performed by A.R., C.H., J.P., D.A.E., K.S., M.F., S.L., M.S., B.P., and G.H. The work for important intellectual content was drafted/revised by A.R., C.H., B.P., and G.H.

\section{Data Availability Statement}

All datasets generated during and/or analyzed during the current study are not publicly available but are available from the corresponding author on reasonable request.

\section{References}

1 Whyte MP. Hypophosphatasia: aetiology, nosology, pathogenesis, diagnosis and treatment. Nat Rev Endocrinol. 2016 Feb;12(4):233-46.

2 Whyte MP, Madson KL, Phillips D, Reeves AL, McAlister WH, Yakimoski A, et al. Asfotase alfa therapy for children with hypophosphatasia. JCI Insight. 2016 Jun;1(9):e85971.

3 Narisawa S, Fröhlander N, Millán JL. Inactivation of two mouse alkaline phosphatase genes and establishment of a model of infantile hypophosphatasia. Dev Dyn. 1997 Mar; 208(3):432-46.

4 Hofmann C, Liese J, Schwarz T, Kunzmann S, Wirbelauer J, Nowak J, et al. Compound heterozygosity of two functional null mutations in the ALPL gene associated with deleterious neurological outcome in an infant with hypophosphatasia. Bone. 2013 Jul;55(1):150-7.
5 Demirbilek H, Alanay Y, Alikaşifoğlu A, Topçu M, Mornet E, Gönç N, et al. Hypophosphatasia presenting with pyridoxine-responsive seizures, hypercalcemia, and pseudotumor cerebri: case report. J Clin Res Pediatr Endocrinol. 2012 Mar;4(1):34-8.

6 Lia-Baldini AS, Muller F, Taillandier A, Gibrat JF, Mouchard M, Robin B, et al. A molecular approach to dominance in hypophosphatasia. Hum Genet. 2001 Jul;109(1):99108.

7 Orimo H, Goseki-Sone M, Sato S, Shimada T. Detection of deletion 1154-1156 hypophosphatasia mutation using TNSALP exon amplification. Genomics. 1997 Jun;42(2):364-6.

8 Whyte MP. Physiological role of alkaline phosphatase explored in hypophosphatasia. Ann N Y Acad Sci. 2010;1192(1):190-200.
9 Rader BA. Alkaline phosphatase, an unconventional immune protein. Front Immunol. 2017 Aug;8:897.

10 Brun-Heath I, Ermonval M, Chabrol E, Xiao J, Palkovits M, Lyck R, et al. Differential expression of the bone and the liver tissue nonspecific alkaline phosphatase isoforms in brain tissues. Cell Tissue Res. 2011 Mar; 343(3):521-36.

11 Baumgartner-Sigl S, Haberlandt E, Mumm S, Scholl-Bürgi S, Sergi C, Ryan L, et al. Pyridoxine-responsive seizures as the first symptom of infantile hypophosphatasia caused by two novel missense mutations (c.677T $>\mathrm{C}$, p.M226T; c.1112C > T, p.T371I) of the tissuenonspecific alkaline phosphatase gene. Bone. 2007 Jun;40(6):1655-61. 
12 Taketani T. Neurological symptoms of hypophosphatasia. Neuronal tissue-nonspecific alkaline phosphatase. Dorddrecht: Spinger Science+Business Media; 2015. p. 309-22.

13 Sebastián-Serrano Á, de Diego-García L, Martínez-Frailes C, Ávila J, Zimmermann H, Millán JL, et al. Tissue-nonspecific alkaline phosphatase regulates purinergic transmission in the central nervous system during development and disease. Comput Struct Biotechnol J. 2015;13:95-100.

14 Fonta C, Barone P, Rodriguez Martinez L, Négyessy L. Rediscovering TNAP in the brain: a major role in regulating the function and development of the cerebral cortex. In: Fonta C, Négyessy L, editors. Neuronal Tissue-Nonspecific Alkaline Phosphatase (TNAP). Dordrecht: Springer Netherlands; 2015. p. 85106.

15 Hanics J, Barna J, Xiao J, Millán JL, Fonta C, Négyessy L. Ablation of TNAP function compromises myelination and synaptogenesis in the mouse brain. Cell Tissue Res. 2012 Aug; 349(2):459-71.

16 Cruz T, Gleizes M, Balayssac S, Mornet E, Marsal G, Millán JL, et al. Identification of altered brain metabolites associated with TNAP activity in a mouse model of hypophosphatasia using untargeted NMR-based metabolomics analysis. J Neurochem. 2017 Mar;140(6): 919-40.

17 Kermer V, Ritter M, Albuquerque B, Leib C, Stanke M, Zimmermann H. Knockdown of tissue nonspecific alkaline phosphatase impairs neural stem cell proliferation and differentiation. Neurosci Lett. 2010 Nov;485(3): 208-11.

18 Díez-Zaera M, Díaz-Hernández JI, Hernández-Álvarez E, Zimmermann H, DíazHernández M, Miras-Portugal MT. Tissuenonspecific alkaline phosphatase promotes axonal growth of hippocampal neurons. Mol Biol Cell. 2011 Jan;22(7):1014-24.
19 de Roo MGA, Abeling NGGM, Majoie CB, Bosch AM, Koelman JHTM, Cobben JM, et al. Infantile hypophosphatasia without bone deformities presenting with severe pyridoxineresistant seizures. Mol Genet Metab. 2014 Mar;111(3):404-7.

20 Fukazawa M, Tezuka J, Sasazuki M, Masumoto N, Baba H, Doi T, et al. Infantile hypophosphatasia combined with vitamin B6-responsive seizures and reticular formation lesions on magnetic resonance imaging: a case report. Brain Dev. 2018;40(2):140-4.

21 Négyessy L, Xiao J, Kántor O, Kovács GG, Palkovits M, Dóczi TP, et al. Layer-specific activity of tissue non-specific alkaline phosphatase in the human neocortex. Neuroscience. 2011 Jan;172:406-18.

22 Waymire KG, Mahuren JD, Jaje JM, Guilarte TR, Coburn SP, MacGregor GR. Mice lacking tissue non-specific alkaline phosphatase die from seizures due to defective metabolism of vitamin B-6. Nat Genet. 1995 Sep;11(1):4551.

23 van der Stoep N, Onkenhout W, Prins S, Struys E, Jakobs C, Peeters-Scholte C. Comments on "Infantile hypophosphatasia without bone deformities presenting with severe pyridoxine-resistant seizures' in molecular genetics and metabolism" 2014 Mar;111(3): 404-7 by M.G. de Roo, N.G. Abeling, C.B. Majoie, A.M. Bosch, J.H. Koelman, J.M. Cobben, M. Duran, B.T. Poll-The. Mol Genet Metab Rep. 2014 May;1:220-2.

24 Mauro SD, Manes T, Hessle L, Kozlenkov A, Pizauro JM, Hoylaerts MF, et al. Kinetic characterization of hypophosphatasia mutations with physiological substrates. J Bone Miner Res. 2002;17(8):1383-91.

25 Kishnani PS, Rush ET, Arundel P, Bishop N, Dahir K, Fraser W, et al. Monitoring guidance for patients with hypophosphatasia treated with asfotase alfa. Mol Genet Metab. 2017 Sep;122(1):4-17.
26 Béthenod M, Cotte MF, Collombel C, Fréderich A, Cotte J. [Neonatal discovery of hypophosphatasia. Bone improvement. Fatal convulsant encephalopathy]. Ann Pediatr (Paris). 1967;14(12):835-41.

27 Balasubramaniam S, Bowling F, Carpenter K, Earl J, Chaitow J, Pitt J, et al. Perinatal hypophosphatasia presenting as neonatal epileptic encephalopathy with abnormal neurotransmitter metabolism secondary to reduced cofactor pyridoxal-5'-phosphate availability. J Inherit Metab Dis. 2010;33 Suppl 3:S25-33.

28 Whyte MP, Greenberg CR, Salman NJ, Bober MB, McAlister WH, Wenkert D, et al. Enzyme-replacement therapy in life-threatening hypophosphatasia. N Engl J Med. 2012; 366(10):904-13.

29 Belachew D, Kazmerski T, Libman I, Goldstein AC, Stevens ST, Deward S, et al. Infantile hypophosphatasia secondary to a novel compound heterozygous mutation presenting with pyridoxine-responsive seizures. JIMD Rep. 2013;11:17-24.

30 Leung ECW, Mhanni AA, Reed M, Whyte MP, Landy H, Greenberg CR. Outcome of perinatal hypophosphatasia in manitoba mennonites: a retrospective cohort analysis. JIMD Rep. 2013;11:73-8.

31 Güzel Nur B, Çelmeli G, Manguoğlu E, Soyucen E, Bircan I, Mıhçı E. Pyridoxine-Responsive Seizures in Infantile Hypophosphatasia and a Novel Homozygous Mutation in ALPL Gene. J Clin Res Pediatr Endocrinol. 2016; 8(3):360-4.

32 Oyachi M, Harada D, Sakamoto N, Ueyama K, Kondo K, Kishimoto K, et al. A case of perinatal hypophosphatasia with a novel mutation in the ALPL gene: clinical course and review of the literature. Clin Pediatr Endocrinol. 2018;27(3):179-86. 